\title{
A battery model that enables consideration of realistic anisotropic environment surrounding an active material particle and its application
}

\author{
Xianke Lin and Wei Lu* \\ Department of Mechanical Engineering, University of Michigan, Ann Arbor, Michigan 48109, \\ USA \\ * Corresponding author. Tel.: +1 734647 7858; fax: +1 734647 3170; E-mail addresses: \\ weilu@umich.edu (Wei Lu)
}




\begin{abstract}
This paper proposes a model that enables consideration of the realistic anisotropic environment surrounding an active material particle by incorporating both diffusion and migration of lithium ions and electrons in the particle. This model makes it possible to quantitatively evaluate effects such as fracture on capacity degradation. In contrast, the conventional model assumes isotropic environment and only considers diffusion in the active particle, which cannot capture the effect of fracture since it would predict results contradictory to experimental observations. With the developed model we have investigated the effects of active material electronic conductivity, particle size, and State of Charge (SOC) swing window when fracture exists. The study shows that the low electronic conductivity of active material has a significant impact on the lithium ion pattern. Fracture increases the resistance for electron transport and therefore reduces lithium intercalation/deintercalation. Particle size plays an important role in lithium ion transport. Smaller particle size is preferable for mitigating capacity loss when fracture happens. The study also shows that operating at high SOC reduces the impact of fracture.
\end{abstract}

Keywords: lithium; particle; fracture; degradation; conductivity; model 


\section{Introduction}

Growing environmental issues coupled with concerns about global crude supplies have stimulated research into Electric vehicles (EV) and hybrid electric vehicles (HEV) [1-4]. Compared to conventional vehicles, HEVs and EVs offer major benefits of improved fuel economy and reduced emissions. Lithium ion battery is one of the most important components of EVs and HEVs, yet it faces a major challenge of capacity degradation or aging during operation [5-9]. Fracture in the active material particle is an important mechanism for capacity degradation. For instance, local fractures have been observed in the Lithium Manganese Oxide (LMO) materials for lithium ion batteries [10]. Figure 1 shows the comparison of SEM images between a fresh LMO cathode and a cycled cathode. Fractures in particles are clearly visible after about 1000 cycles. The cause of fracture in the LMO particle is the high stress induced by lithium intercalation/deintercalation, especially at high charge/discharge rates. A substantial amount of work has gone into understanding stress generation and fracture propagation in active material particles. For instance, Zhang et al. [11] developed a three dimensional stress model to study intercalation-induced stress in cathode particles. They investigated the lithium intercalationinduced stress at different particle sizes and aspect ratios, and suggested that smaller sizes are desirable for reducing intercalation-induced stress during cycling. With an extended finite element method, Zhu et al.[12] analyzed the effects of current density, particle size and particle aspect ratio on fracture propagation. While these studies have highlighted stress generation and fracture propagation in a particle under various conditions, a relationship between fracture and the degree of capacity degradation that it causes has not been established. The impact of fracture on capacity degradation during discharge/charge cycles remains to be investigated. 
Fracture can affect the performance of an electrode in several ways. Take the LMO material as an example. Fracture increases the surface area exposed to the electrolyte, therefore increasing the side reaction rate and $\mathrm{Mn}^{2+}$ dissolution $[13,14]$. The increased surface area might be beneficial for lithium intercalation/deintercalation due to increased contact area with the electrolyte. However, fracture also leads to the loss of electrical contact which contributes to capacity degradation. In order to understand the impact of fracture on cell performance, this paper develops a single particle fracture model considering both diffusion and migration of lithium ions and electrons in the particle, with a LMO particle as a representative system. Unlike the conventional model where a particle is assumed isotropic, here we consider the realistic anisotropic environment typically found surrounding a particle, such as inhomogenous carbon black distribution on the particle surface. The conventional model only considers diffusion in a particle, which cannot capture the impact of fracture. We argue that it is necessary to consider both diffusion and migration of both lithium ions and electrons in the particle. We have used the model to quantify capacity degradation associated with fracture under various operational conditions. The study has shown the impact of material electronic conductivity, State of Charge (SOC) swing window, and particle size on capacity. Optimal design and operational guidelines have been obtained to suppress the impact of fracture on capacity degradation.

\section{Modeling of a particle with anisotropic surrounding environment and internal fracture}

\subsection{Composition and micro-structure}



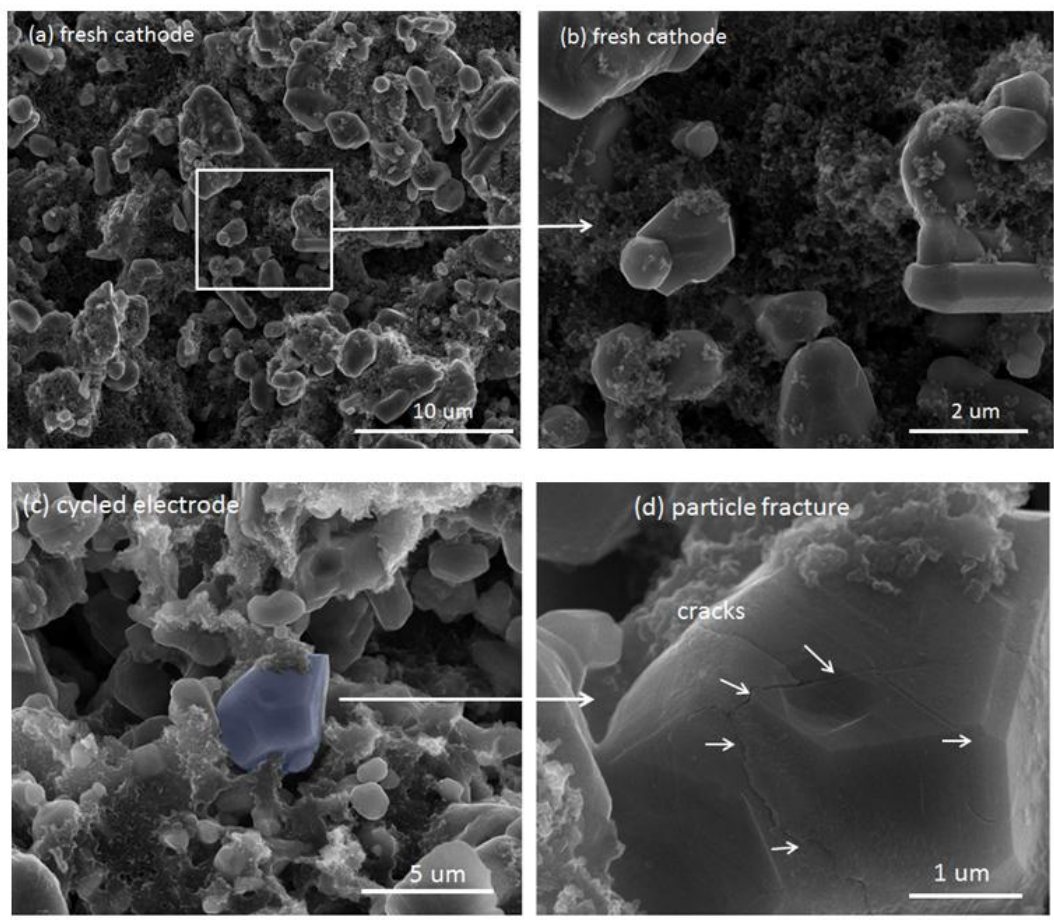

Figure 1. (a),(b) SEM images of a fresh LMO cathode. (c),(d) SEM images of a cathode after 1200 charge/discharge cycles. Fractures are clearly visible. Fractured particles often have parts which are surrounded by fracture surfaces. These parts are almost isolated from the rest of the particles. The composite electrode consists of 5\% carbon black, 5\% PVDF, and 90\% LMO from Sigma-Aldrich.

A typical LMO cathode is made by mixing 5\% carbon black, 90\% LMO active material and 5\% PVDF binder together. As shown in Fig. 1 (a), (b), carbon black is not distributed evenly on the surface of each particle. Instead, a considerable portion of carbon black aggregates inbetween LMO particles and form a conductive network. Only a fraction of carbon black is in contact with LMO particles. The surface of a LMO particle is not fully covered by carbon black, and part of the surface area is exposed to the electrolyte. The carbon black connected to particles forms bridges for electron transport between the particles and the current collector and inbetween the particles. The surface area exposed to the electrolyte is available for lithium ion intercalation/deintercalation. Figure 2 (a) illustrates the distribution of carbon black and the 
electron pathways. After cycling, fracture develops as shown in Fig. 1 (c) and (d). Fractured particles often have parts which are surrounded by fracture surfaces. These parts are almost isolated from the rest of the particles as shown in Fig. 1 (d).

(a) fresh cathode

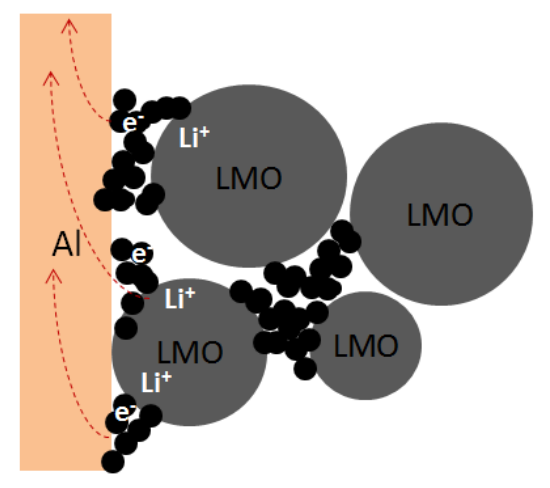

(b) diffusion-only model

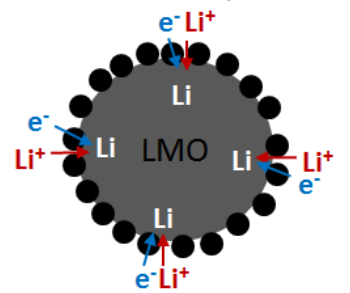

(c) diffusion \& migration model

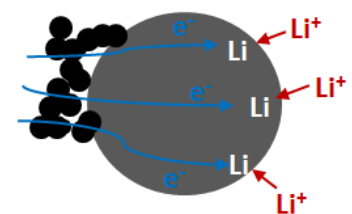

Figure 2. (a) Sketch of the particle structures in the composite electrode. Due to carbon black aggregation, only a small percentage of carbon black is in contact with the particle, and the contact only covers a fraction of the particle surface. (b) Diffusion-only model assumes that the carbon black is uniformly distributed on the particle surface and therefore electrons and lithium ions react uniformly, and only diffusion governs the lithium transport inside the particle. (c) We consider that carbon black is not distributed evenly on the surface, and that the mass transport of lithium ions and electrons is controlled by both migration and diffusion. Therefore, the fractureinduced electrical isolation or increase in electrical resistance can be captured.

In the conventional model [15], the lithium transport in the particle is modeled by diffusion only as illustrated in Fig. 2 (b). Diffusion-only model assumes that the carbon black is uniformly distributed on the particle surface, and therefore electrons and lithium ions react uniformly on the surface so that only diffusion governs the lithium transport inside the particle. The diffusion-only model has a uniform flux on the particle surface. Therefore, larger particle surface facilitates lithium ion and electron reaction, and increases lithium diffusion. A diffusiononly model predicts that fracture of a particle is beneficial since it increases the surface area and 
therefore increases the capacity for any given C-rate. However, this prediction is contradictory to experimental observations [16]. We have found that a major limitation of the diffusional-only model is that it cannot capture electrical isolation. In our proposed diffusion and migration model illustrated in Fig. 2 (c), we consider that carbon black is not distributed evenly on the surface, and that the mass transport of lithium ions and electrons is controlled by both migration and diffusion. Therefore, the fracture-induced electrical isolation or increase in electrical resistance can be captured.

The process can be understood in the following. Consider a $\mathrm{LiMn}_{2} \mathrm{O}_{4} / \mathrm{Li}$ half-cell. When it is being charged, electrons are transferred out of the cathode. The carbon black network provides the pathway for electron transport. Once the electrons in the LMO particles are out, due to charge neutrality, lithium ions are deintercalated out of the particles as well. When the cell is being discharged, electrons are injected into the cathode current collector from the external circuit and transferred to the LMO particles through the carbon black network. The lithium ions in the electrolyte are also intercalated into the particles due to charge neutrality.

We consider a particle in a $\mathrm{LiMn}_{2} \mathrm{O}_{4} / \mathrm{Li}$ half-cell as shown in Fig. 3. The model is constructed in COMSOL and consists of 4 domains: carbon black (domain 1), LMO particle (domain 2), electrolyte and separator (domain 3), and lithium metal anode (domain 4). In the following we use subscripts 1, 2, 3, 4 to denote these domains, and use subscripts (12), (23), (34) to denote the interfaces between carbon black and the LMO particle, the LMO particle and electrolyte, and electrolyte and the Li metal anode, respectively. When the cell is being charged, the current flux is added to the carbon black surface. The electrons are transferred out of the LMO particle through the carbon black, and lithium ions are deintercalated into the electrolyte. Lithium ions in the electrolyte are transferred to the anode side and deposited on the surface of 
lithium metal. Here we consider a case that the carbon black distributes mainly on one side of the particle. Figure 3 (b) shows the carbon black distribution generated by using a Matlab random function. The many fine carbon black particles of domain 1, domain 2 and domain 3, are all modeled with finite element meshes. Domain 4 is treated as a boundary with zero voltage.
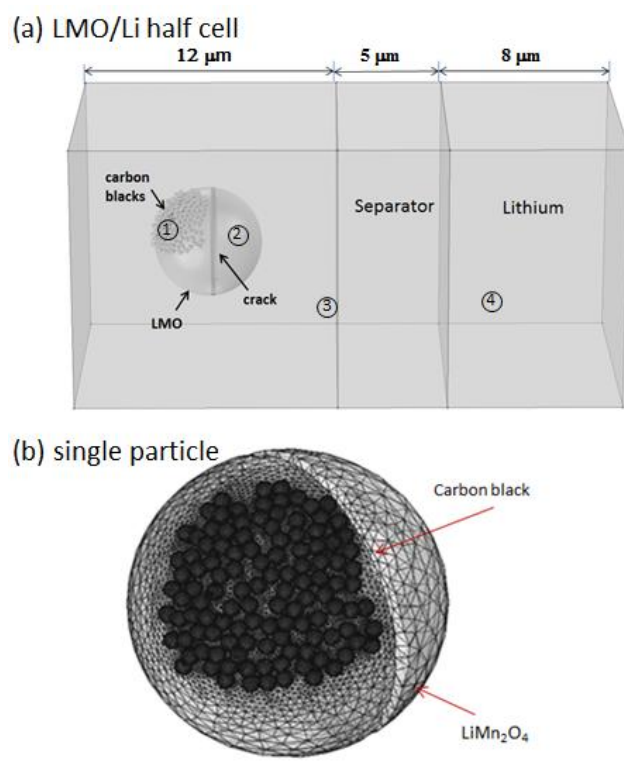

Figure 3. (a) A LMO/Li half-cell that includes carbon black (domain1), LMO particle (domain2), electrolyte and separator (domain3), and lithium metal anode (domain4). (b) Meshed geometry of a LMO particle with attached fine carbon black particles.

The typical mass ratio between carbon black and LMO in a cell is 5\%:90\% or 1: 18 . Due to carbon black aggregation, only a fraction of the fine carbon black particles are in contact with the surface of the LMO particle. Based on SEM images of the LMO electrode, we found that by average about $40 \%$ of carbon black is attached to the surface. Therefore, the mass ratio between the attached carbon black particles and the LMO particle is taken to $1: 45$ in our simulation. 
We developed a MATLAB program to generate the carbon black distribution. The LMO particle, which has a radius of $r$, is located at $(0,0,0)$ in the $x y z$ coordinate system. First, the number of carbon black particles is calculated by the size of carbon black particles, the size of the LMO particle and their mass ratio (1:45). In our study, the number of carbon black particles varies from 49 to 118 in different cases of particle sizes. Then the MATLAB random function is called to generate a random position $(x, y, 0)$ within a circle centered at $(-r, 0,0)$ with a radius of $0.4 r$ in the $x y$-plane. We will discard the position $(x, y, 0)$ if its distance to $(0,0,0)$ is larger than the radii of the LMO particle and of the carbon black particle added together. Otherwise we calculate the corresponding $z$ coordinate by the distance between particle centers, so that the carbon black particle centered at $(x, y, z)$ contacts the LMO particle. We further check the position $(x, y, z)$ and discard it if (1) the carbon black particle at this position overlaps with other carbon black particles already on the LMO particle surface; or (2) the gap between the carbon black particle at this position and the nearest carbon black particle already on the LMO particle surface is larger than a small threshold, such as $5 \mathrm{~nm}$. Otherwise this position is recorded. This checking process ensures that there is no overlap of carbon black particles, and there is no isolated carbon black particle so that the carbon black particles connect to form one network. The random position generation process continues until reaching the required number of carbon black particles. The positions of all carbon blacks are then imported to COMSOL by a MATLAB script. In our study, three carbon black sizes with diameters of 300nm, $250 \mathrm{~nm}, 70 \mathrm{~nm}$ have been used respectively for the LMO particle sizes of $8 \mu \mathrm{m}, 5 \mu \mathrm{m}$ and $1 \mu \mathrm{m}$.

\subsection{Particle model considering coupled diffusion and migration with anisotropic environment}


Carbon black has high electronic conductivity and the mobile species are electrons. The flux density of electrons in the carbon black network is governed by Ohm's law as

$$
\begin{gathered}
\mathbf{E}_{1}=-\nabla \phi_{1} \\
\mathbf{i}_{1}=\sigma_{1} \mathbf{E}_{1}
\end{gathered}
$$

where $\phi_{1}$ is the electric potential in the carbon black, $\mathbf{E}_{1}$ is the electric field, $\mathbf{i}_{1}$ is the current density or the negative flux density of electrons, and $\sigma_{1}$ is the electronic conductivity. In domain 1 the potential field is solved by $\nabla \cdot \mathbf{i}_{1}=0$. The current density boundary condition is applied on the carbon black surface. The total applied current is calculated by the capacity of the LMO particle and the C-rate, which gives the current density after dividing the surface area of carbon black.

Electron flux can pass through the interface between the LMO particle and carbon black. There is no electron transport across the interface between carbon black and the electrolyte, across the interface between the LMO particle and the electrolyte, or across the interface between the electrolyte and lithium metal.

The electrochemical reaction at the LMO/electrolyte interface can be expressed by

$$
\mathrm{Li}^{+}+\mathrm{Mn}_{2} \mathrm{O}_{4}+\mathrm{e}^{-} \leftrightarrow \mathrm{LiMn}_{2} \mathrm{O}_{4}
$$

The lithium intercalation/deintercalation rate is governed not only by lithium ion concentrations in both the electrolyte and the LMO particle, but also by the local potential difference between the electrolyte and the LMO particle. The following Butler-Volmer equation is used to model the lithium intercalation/deintercalation rate:

$$
\begin{gathered}
i_{23}=i_{23,0}\left(\exp \left(\frac{\alpha_{a} F}{R T} \eta_{23}\right)-\exp \left(-\frac{\alpha_{c} F}{R T} \eta_{23}\right)\right) \\
\eta_{23}=\phi_{2}-\phi_{3}-U
\end{gathered}
$$


where $i_{23}$ is the reaction current density between LMO and the electrolyte, $i_{23,0}$ is the exchange current density, $\alpha_{a}$ is the anodic charge transfer coefficient, $\alpha_{c}$ is the cathodic charge transfer coefficient, $F$ is the Faraday constant, $R$ is the gas constant, $T$ is the absolute temperature, $\phi_{2}$ is the electric potential in the LMO particle, $\phi_{3}$ is the electric potential in the electrolyte, and $U$ is the LMO cathode equilibrium potential.

In the LMO particle, the mobile species are lithium ions and electrons. The $\mathrm{Mn}_{2} \mathrm{O}_{4}$ host structure is stationary. Yamamura et al. [17] conducted an in situ measurement of $\mathrm{LiMn}_{2} \mathrm{O}_{4}$ conductivity at different SOCs. Their measurements showed an almost negative linear dependence of conductivity on voltage, ranging between $2.4 \times 10^{-3} \mathrm{~S} \mathrm{~m}^{-1}$ at $3.5 \mathrm{~V}$ and $1.3 \times 10^{-3} \mathrm{~S}$ $\mathrm{m}^{-1}$ at $4.2 \mathrm{~V}$. The conductivity at high voltage, when there is little intercalated lithium, indicates that there are mobile electrons in $\mathrm{Mn}_{2} \mathrm{O}_{4}$. As lithium ions intercalate into the LMO particle, the mobile electrons in the particle are increased. Based on charge neutrality, the total amount of mobile electrons is equal to the sum of lithium ions and the amount of mobile electrons provided by the host structure $\mathrm{Mn}_{2} \mathrm{O}_{4}$. The two sources of mobile electrons can be expressed by

$$
\begin{gathered}
\mathrm{Li} \leftrightarrow \mathrm{Li}^{+}+\mathrm{e}^{-} \\
\mathrm{Mn}_{2} \mathrm{O}_{4} \leftrightarrow\left(\mathrm{Mn}_{2} \mathrm{O}_{4}\right)^{x+}+x \mathrm{e}^{-}
\end{gathered}
$$

The fluxes of negatively and positively charged species in the particle are governed by the Nernst-Planck equation. This equation is obtained by combining Ohm's law and the diffusion equation to keep charge conservation and mass conservation. The flux density of each species is not only controlled by the concentration gradient but also by the potential gradient:

$$
\mathbf{J}_{2, i}=-D_{2, i} \nabla c_{2, i}-z_{2, i} u_{m, i} F c_{2, i} \nabla \phi_{2}
$$


where $\mathbf{J}_{2, i}$ represents the flux density of the species $i$, which can be 'e ' (electron), 'Li' (lithium ion) or ' $\mathrm{Mn}_{2} \mathrm{O}_{4}{ }^{\prime}\left(\left(\mathrm{Mn}_{2} \mathrm{O}_{4}\right)^{x+}\right.$ ion). In the equations $D_{2, i}$ is the diffusion coefficient of species $i$, $c_{2, i}$ is the concentration, $z_{2, i}$ is the charge number, and $u_{m, i}$ is the mobility. Because $\left(\mathrm{Mn}_{2} \mathrm{O}_{4}\right)^{x+}$ is stationary, we assign a very small diffusion coefficient to it so that its flux is negligible.

The mobility of species $i$ is related to its diffusion coefficient by the Einstein relation,

$$
u_{m, i}=\frac{D_{2, i}}{R T}
$$

The mass conservation of each species is given by

$$
\frac{\partial c_{2, i}}{\partial t}=-\nabla \cdot \mathbf{J}_{2, i}=\nabla \cdot\left(D_{2, i} \nabla c_{2, i}+z_{2, i} u_{m, i} F c_{2, i} \nabla \phi_{2}\right)
$$

The charge conservation is given by

$$
\sum_{i} z_{2, i} c_{2, i}=0
$$

Note that while $\left(\mathrm{Mn}_{2} \mathrm{O}_{4}\right)^{x+}$ is stationary itself, it affects the transport of mobile species, $\mathrm{e}^{-}$and $\mathrm{Li}^{+}$, by charge conservation.

The current in the particle is given by

$$
\mathbf{i}_{2}=F \sum_{i} z_{2, i} \mathbf{J}_{2, i}
$$

In domain 2 the concentration fields $c_{2, \mathrm{Li}}, c_{2, \mathrm{e}}$ and the potential field $\phi_{2}$ are solved by Eqs. (10) and $\nabla \cdot \mathbf{i}_{2}=0$. The $c_{2, \mathrm{Mn}_{2} \mathrm{O}_{4}}$ field is solved by charge conservation. At the interface between carbon black and the LMO particle, the current is continuous or $\mathbf{n} \cdot \mathbf{i}_{1}=\mathbf{n} \cdot \mathbf{i}_{2}$ where $\mathbf{n}$ is the normal vector of the particle surface, and $\mathbf{n} \cdot \mathbf{J}_{2, \mathrm{Li}}=0$ since lithium ions cannot transport into the 
carbon black domain. At the interface between the LMO particle and electrolyte, the flux density of lithium ion is given by $\mathbf{n} \cdot \mathbf{J}_{2, \mathrm{Li}}=i_{23} / F$, while the flux density of electron is $\mathbf{n} \cdot \mathbf{J}_{2, \mathrm{e}}=0$ since electron cannot transport into the electrolyte.

The electrolyte ( $\mathrm{LiPF}_{6} 1 \mathrm{M}$ EC/DMC) is assumed to be binary. Charge neutrality is satisfied by keeping the concentration of lithium ions equal to the concentration of negatively charged species. Based on the concentrated solution theory [18], the flux density of the positively charged ions and negatively charged ions can be expressed by the gradient of the electrochemical potential. The gradient of the electrochemical potential can be decomposed into gradients of the salt concentration and the electrical potential $[19,20]$. The flux density of the lithium ions and the negatively charged species can be expressed in the following equations. The detailed derivation is included in the appendix.

$$
\begin{gathered}
\mathbf{J}_{3,+}=\left(-D_{3} \frac{c_{T}}{c_{0}}+\frac{2 k_{3} t_{-}^{0} t_{+}^{0} R T}{c_{3,+} z_{3,+} F^{2}}\right)\left(1+\frac{d \ln f_{ \pm}}{d \ln c_{3}}\right) \nabla c_{3,+}-\frac{k_{3} t_{+}^{0}}{z_{3,+} F} \nabla \phi_{3} \\
\mathbf{J}_{3,-}=\left(-D_{3} \frac{c_{T}}{c_{0}}+\frac{2 k_{3} t_{-}^{0} t_{-}^{0} R T}{c_{3,-} z_{3,-} F^{2}}\right)\left(1+\frac{d \ln f_{ \pm}}{d \ln c_{3}}\right) \nabla c_{3,-}-\frac{k_{3} t_{-}^{0}}{z_{3,-} F} \nabla \phi_{3} \\
c_{T}=\sum_{i} c_{3, i}+c_{0} \\
\frac{c_{3,+}}{v_{3,+}}=\frac{c_{3,-}}{v_{3,-}}=c_{3}
\end{gathered}
$$

where $\mathbf{J}_{3,+}$ and $\mathbf{J}_{3,-}$ are the flux densities of lithium ion and the negatively charged ion in the electrolyte, $v_{3,+}$ and $v_{3,-}$ are the numbers of cations and anions into which a molecule of the electrolyte dissociates, $D_{3}$ and $k_{3}$ are the diffusivity and conductivity of the electrolyte, $c_{T}$ is the total concentration including the solvent, where $i$ denotes the + and - species, $c_{0}$ is the solvent 
concentration, $t_{+}^{0}$ and $t_{-}^{0}$ are the transference numbers of lithium ion and the negatively charged ion in the electrolyte, $c_{3}$ is the electrolyte concentration, $c_{3,+}$ and $c_{3,-}$ are the concentrations of lithium ion and the negatively charged ion, $z_{3,+}$ and $z_{3,-}$ are charge numbers, $f_{ \pm}$is the electrolyte activity coefficient, $c_{3}$ is the electrolyte concentration, and $\phi_{3}$ is the electric potential of the electrolyte.

Similar as Eqs. (10) - (12), we can write a set of equations for the electrolyte: $\partial c_{3, i} / \partial t=-\nabla \cdot \mathbf{J}_{3, i}, \sum_{i} z_{3, i} c_{3, i}=0, \mathbf{i}_{3}=F \sum_{i} z_{3, i} \mathbf{J}_{3, i}$, and $\nabla \cdot \mathbf{i}_{3}=0$. In domain 3 the concentration field $c_{3,+}$ and the potential field $\phi_{3}$ are solved by these differential equations. The $c_{3,-}$ field is solved by charge conservation. At the interface between the LMO particle and electrolyte, the flux density of lithium ion is given by $\mathbf{n} \cdot \mathbf{J}_{3,+}=i_{23} / F$ while the flux density of negatively charged ion is $\mathbf{n} \cdot \mathbf{J}_{3,-}=0$.

On the surface of the lithium metal anode, lithium deposition and dissolution occurs during charging and discharging. The reaction rate is controlled by the over potential between the anode and the electrolyte as well as lithium ion concentration in the electrolyte by

$$
\begin{gathered}
i_{43}=i_{43,0}\left(\exp \left(\frac{\alpha_{a, \mathrm{Li}} F}{R T} \eta_{43}\right)-\exp \left(-\frac{\alpha_{c, \mathrm{Li}} F}{R T} \eta_{43}\right)\right) \\
\eta_{43}=\phi_{4}-\phi_{3}
\end{gathered}
$$

where $i_{43}$ is the reaction current density between lithium metal and the electrolyte, $i_{43,0}$ is the exchange current density, $\alpha_{a, \mathrm{Li}}$ is the anodic charge transfer coefficient, $\alpha_{c, \mathrm{Li}}$ is the cathodic charge transfer coefficient, and $\phi_{4}$ is the electric potential in the lithium metal which is assigned zero as the boundary condition. 
At the interface between electrolyte and the Li metal anode, the flux density of lithium ion is given by $\mathbf{n} \cdot \mathbf{J}_{3,+}=-i_{43} / F$, where $\mathbf{n}$ is the normal vector of the interface pointing from domain 3 to domain 4 . The negative sign is because $i_{43}$ gives the lithium ion flux density from the anode to the electrolyte. The flux density of negatively charged ion at the interface between electrolyte and the $\mathrm{Li}$ metal anode is $\mathbf{n} \cdot \mathbf{J}_{3,-}=0$.

Relevant material properties for carbon black, $\mathrm{LiMn}_{2} \mathrm{O}_{4}, \mathrm{EC} / \mathrm{DMC} 1 \mathrm{M} \mathrm{LiPF} 6$ electrolyte and lithium metal are listed in Table 1.

\section{Results and discussions}

\subsection{Model validation against conductivity experiment}

We first performed a simulation to validate the model against the experimental results of pure LMO material electronic conductivity as a function of SOC. To facilitate the extraction of conductivity information and experimental comparison, we constructed a cylindrical particle as shown in Fig. 4. Note that both the simulation and the experiment here were conducted on the pure LMO material, not on a composite LMO electrode. There is no carbon black in the conductivity simulation and measurement. During the electronic conductivity measurement, a given electron flux enters one end of the cylinder along the axial direction and flows out of the cylinder at the other end. The electronic conductivity is calculated by

$$
k_{2}=\frac{I L}{U_{2} A_{2}}
$$

where $k_{2}$ is the conductivity, $I$ is the applied current, $L$ is the distance between the two ends of the cylinder, $U_{2}$ is the voltage difference between the two ends, and $A_{2}$ is cross-section area of the cylinder. In the simulations we used Eqs. (8) - (12) with the boundary condition of an 
applied electron flux at the ends and the initial condition of a given uniform lithium concentration to calculate the potential field and subsequently the voltage difference $U_{2}$. We then used Eq. (19) to obtain the electronic conductivity corresponding to the given lithium concentration and its associated SOC. Simulations were performed at various given lithium concentration conditions to obtain the electronic conductivity as a function of SOC. Note that although there are lithium ions in the solid, the mobile species to carry the continuous steady current are electrons only since there is no electrochemical reaction and no lithium ion moving in or out of the solid. Our simulations show that the concentration in the solid is almost uniform, and the electron flux is driven by the potential gradient. This is consistent with the intended electronic conductivity measurement.

Our simulations show that the electronic conductivity is a linear function of lithium concentration. This is consistent with expectation. Based on charge neutrality, the total amount of mobile electrons is equal to the sum of lithium ions and the amount of mobile electrons provided by the host structure $\mathrm{Mn}_{2} \mathrm{O}_{4}$. The concentration of mobile electrons in $\mathrm{Mn}_{2} \mathrm{O}_{4}$ gives the $c_{2, e}$ at $0 \%$ SOC. Higher lithium concentration leads to proportional increase of mobile electrons and therefore higher conductivity. By fitting the conductivity to $2.4 \times 10^{-3} \mathrm{~S} \mathrm{~m}^{-1}$ at $3.5 \mathrm{~V}(100 \%$ SOC) where $c_{2, e}$ is the mobile electrons in $\mathrm{Mn}_{2} \mathrm{O}_{4}$ plus the lithium concentration at $100 \%$ SOC, and to $1.3 \times 10^{-3} \mathrm{~S} \mathrm{~m}^{-1}$ at $4.2 \mathrm{~V}\left(20 \%\right.$ SOC) where $c_{2, e}$ is the mobile electrons in $\mathrm{Mn}_{2} \mathrm{O}_{4}$ plus the lithium concentration at $20 \%$ SOC, we can obtain two parameters, mobile electrons in $\mathrm{Mn}_{2} \mathrm{O}_{4}$ and $D_{2, \mathrm{e}}$. According to Eq. (11), we have $z_{2, \mathrm{Mn}_{2} \mathrm{O}_{4}} c_{2, \mathrm{Mn}_{2} \mathrm{O}_{4}}=c_{2, e}$ at $0 \%$ SOC. This relation is used to estimate $z_{2, \mathrm{Mn}_{2} \mathrm{O}_{4}}$. Since $\left(\mathrm{Mn}_{2} \mathrm{O}_{4}\right)^{x+}$ is stationary itself and does not diffuse, $z_{2, \mathrm{Mn}_{2} \mathrm{O}_{4}} c_{2, \mathrm{Mn}_{2} \mathrm{O}_{4}}$ always appears together and only affects the charge conservation, therefore the 
separation of $z_{2, \mathrm{Mn}_{2} \mathrm{O}_{4}}$ is not necessary. We estimate its value simply because we use the same form of Eqs. (8) and (10) to solve all species, where $z_{2, \mathrm{i}}$ is a parameter. We assign a very low diffusion coefficient for $\left(\mathrm{Mn}_{2} \mathrm{O}_{4}\right)^{x+}$ to make it stationary.

Figure 4 shows the comparison between our calculated result and the data from experiments [17]. In plotting this figure we have converted the cathode potential to SOC since the original experimental data was expressed as a function of cathode potential. The agreement confirms that our model can well capture the coupled diffusion and migration in the particle, and this coupling is necessary to explain the dependence of conductivity on SOC. The experimental data of conductivity was measured simultaneously during cyclic voltammetry without rest, so the lithium concentration may not be uniform in the sample. This effect could be one of the reasons that the experimental curve is not exactly a straight line. The data at the two ends of the curve are likely to be more consistent with the uniform concentration condition and therefore more accurate.
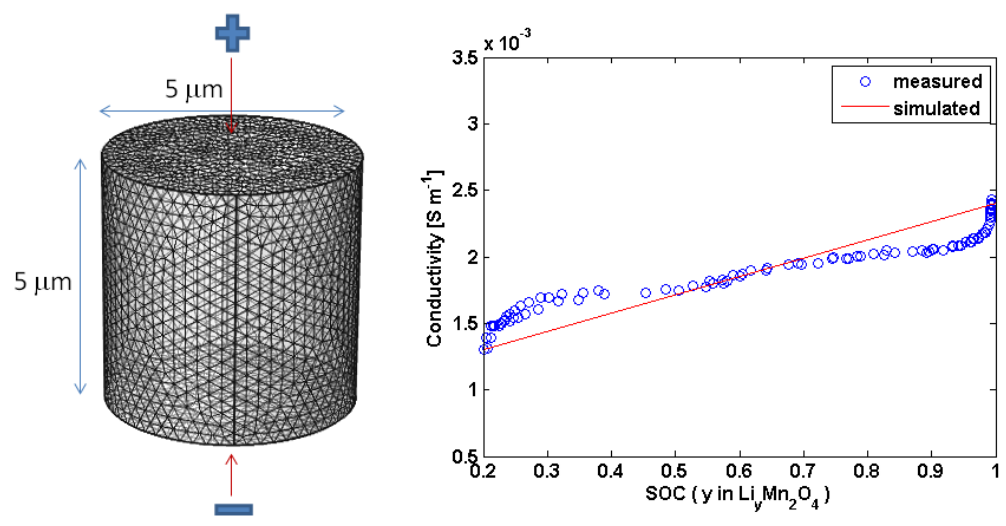

Figure 4. Comparison between the calculation result and the measured data from ref [17].

\subsection{Impact of particle electronic conductivity}


LMO material has a quite low electronic conductivity [17]. In order to understand the impact of electronic conductivity on the lithium ion pattern in LMO particles, we charge the particle at $1 \mathrm{C}$ rate for 1000 seconds under various electronic conductivity conditions to study lithium ion deintercalation. Figure 5 shows the lithium ion distribution in a spherical particle under various electronic conductivity conditions. The values shown are the conductivity at the beginning of charging, since the conductivity will evolve during the charging process, which will be shown later. Figure 5 (a) shows the lithium ion distribution in the LMO particle using the measured conductivity data. Most of the lithium is deintercalated from the side where the carbon black connects to. After increasing the conductivity by 10 times and 100 times, as shown in Fig. 5 (b) and Fig. 5 (c), we can observe more and more symmetric lithium ion distribution. After conductivity is increased by 1000 times as shown in Fig. 5 (d), the lithium pattern is similar to the diffusion-only model. Figure 5 clearly indicates that with high electronic conductivity, lithium intercalation/deintercalation can happen in all directions with same flux density, even though the electrons only transport through a small area on the particle surface that is in direct contact with the carbon black. The fast transport of electrons inside the particle compensates for the anisotropic environment, and lithium intercalation/deintercalation are mainly limited by diffusion from the concentration gradient. With low electronic conductivity, lithium intercalation/deintercalation initiates in the area close to the carbon black. The slow transport of electrons cannot meet the demands for lithium intercalation/deintercalation far away from the carbon black. Therefore, lithium intercalation/deintercalation mainly occurs in the area close to the carbon black. 
(a) $2.41 \times 10^{-3} \mathrm{~S} \mathrm{~m}^{-1}$

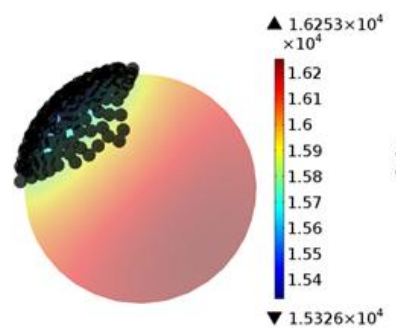

(b) $2.41 \times 10^{-2} \mathrm{~S} \mathrm{~m}^{-1}$

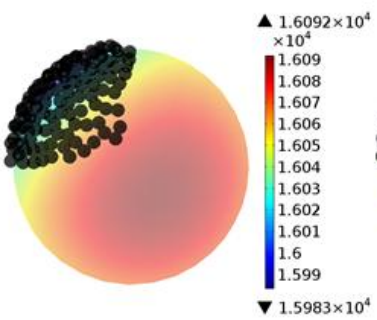

unit: $\mathrm{mol} \mathrm{m}^{-3}$ (c) $2.41 \times 10^{-1} \mathrm{~s} \mathrm{~m}^{-1}$

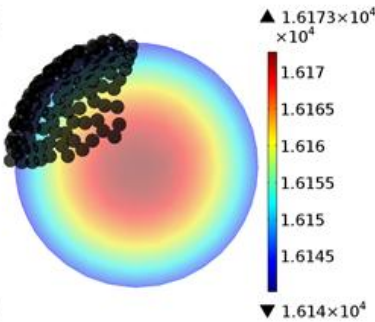

Particle size: $5 \mu \mathrm{m}$ (d) $2.41 \mathrm{~S} \mathrm{~m}^{-1}$

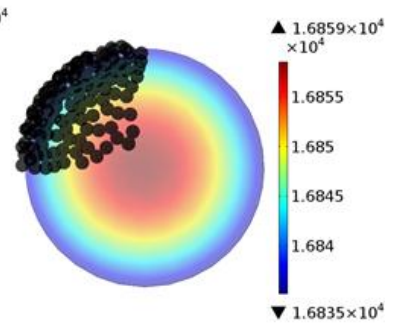

Figure 5. Cross-section view of lithium ion concentration inside a spherical particle under various electronic conductivity conditions. Charge at $1 \mathrm{C}$ rate for 1000 seconds. Values shown are the conductivity at the beginning of charging. Particle diameter: $5 \mu \mathrm{m}$.

\subsection{Impact of fracture on lithium transport}

Fracture increases the surface area in contact with electrolyte, which provides more area for lithium intercalation/deintercalation. A conventional model based on isotropic environment would predict an increase of usable capacity, which is contradictory to experimental observations. We argue that fracture leads to anisotropic transport and reduced conductivity. Consideration of this effect is important to correctly evaluate the impact of fracture. In order to study the impact of fracture on lithium transport in the particle, we introduce a crack in the particle, and charge the particle at $1 \mathrm{C}$ rate until the voltage increases to $4.1 \mathrm{~V}$. Figure 6 (a) shows the lithium ion concentration profile on the surface of the LMO particle without fracture, while Fig. 6 (b) shows the lithium ion concentration profile on the particle surface with fracture. Without fracture, lithium ions deintercalate more in the region where carbon black connects to, but the lithium ion concentration becomes more uniform away from the carbon black region. With a crack, as shown in Fig. 6 (b), the concentration is quite different between the two regions on the left and the right side of the crack. The concentration difference across the crack is about $1679 \mathrm{~mol} \mathrm{~m}^{-3}$. This means that a lot of lithium ions are trapped in the region on the opposite side of the carbon black due to the crack, and cannot deintercalate. 


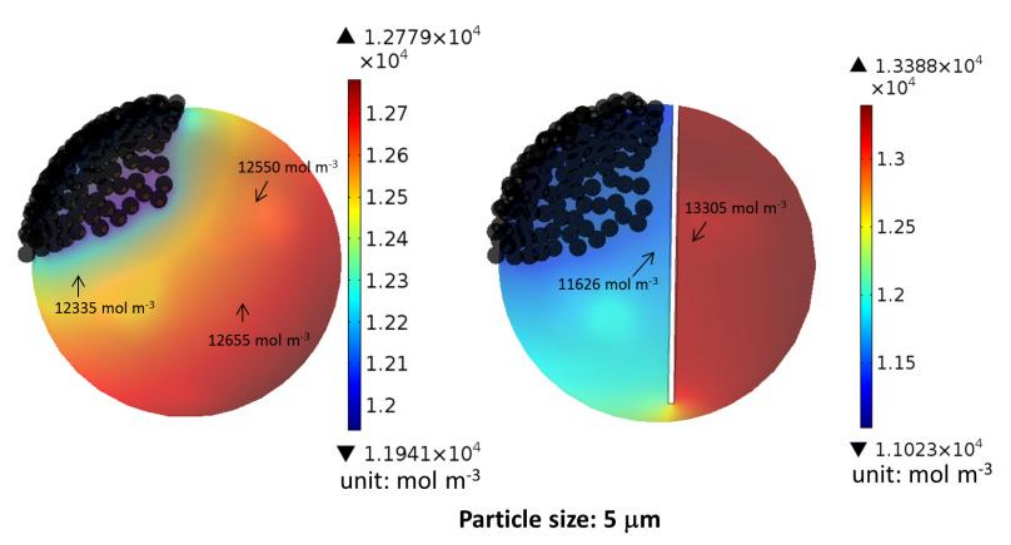

Figure 6. Comparison of lithium ion concentration on particle surfaces with and without fracture. Lithium ions in the region on the opposite side of the carbon black are trapped due to the crack, and cannot deintercalate. Particle diameter: $5 \mu \mathrm{m}$.

\subsection{Impact of particle size}

We have chosen three different particle sizes (in diameter): $1 \mu \mathrm{m}, 5 \mu \mathrm{m}$, and $8 \mu \mathrm{m}$. The mass ratio between carbon black and the LMO particle is kept the same as before. All the particles are charged to $4.1 \mathrm{~V}$. Figure 7 shows the cross-section view of lithium ion concentration inside the particle. We can observe that particle size has a significant impact on lithium transport in the LMO particle when there is fracture. Smaller particles facilitate the transport of lithium ion due to increased specific surface area and reduced internal resistance. The lithium ion concentration difference across the crack decreases with the particle size. When the particle size is $1 \mu \mathrm{m}$ as shown in Fig. 7 (a), the concentration difference across the crack is only $169 \mathrm{~mol} \mathrm{~m}^{-3}$, which is quite small and negligible. However, after increasing the particle size to $5 \mu \mathrm{m}$ as shown in Fig. 7 (b), the concentration difference becomes $1491 \mathrm{~mol} \mathrm{~m}^{-3}$. When the particle size is increased to $8 \mu \mathrm{m}$ as shown in Fig. 7 (c), the concentration difference further increases to 3002 mol m $\mathrm{m}^{-3}$, which is quite significant. These results suggest that smaller particle size is preferable to suppress the impact of fracture. 
(a) particle size: $1 \mu \mathrm{m}$

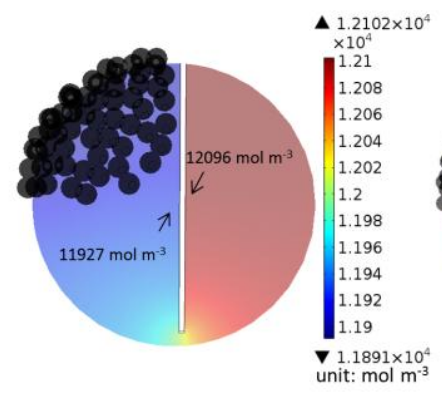

$\Delta c=169 \mathrm{~mol} \mathrm{~m}^{-3}$ (b) particle size: $5 \mu \mathrm{m}$

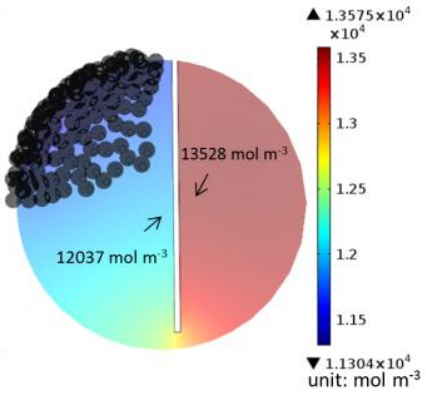

$\Delta c=1491 \mathrm{~mol} \mathrm{~m}^{-3}$ (c) particle size: $8 \mu \mathrm{m}$

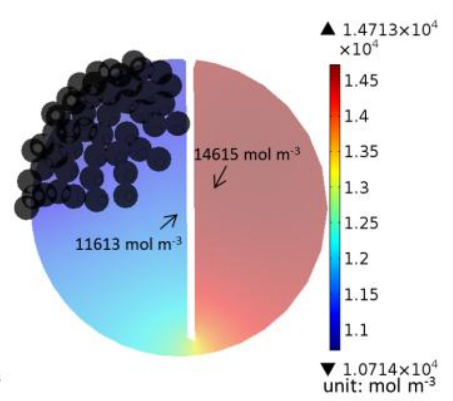

$\Delta c=3002 \mathrm{~mol} \mathrm{~m}^{-3}$

Figure 7. Cross-section view of lithium ion concentration inside particles of various sizes. Smaller particle size reduces the impact from fracture. Particle diameter: $1 \mu \mathrm{m}, 5 \mu \mathrm{m}$, and $8 \mu \mathrm{m}$.

\subsection{Impact of state of charge}

State of charge has a significant impact on the conductivity of the LMO material. In order to understand the impact of SOC on lithium transport, the particle is charged at $1 \mathrm{C}$ rate to different SOCs; the lithium ion concentration profiles inside particles are shown in Fig. 8. We can observe that a lower SOC leads to more concentration difference across the crack, which amplifies the effect of fracture and leads to more capacity degradation.

The comparison between ionic conductivity and electronic conductivity is shown in Fig. 8 (d). Both electronic conductivity and ionic conductivity decrease with time, or SOC. Because carbon black pulls electrons out of the LMO particle during charging, most of the lithium ions are deintercalated from the side that the carbon black connects to. As the lithium ion concentration goes down during charging, the associated ionic conductivity goes down as well. The comparison indicates that the electronic conductivity is smaller than the ionic conductivity. Therefore, lithium ion deintercalation and transport inside the particle is mainly limited by electron transport since the accumulation of electrons makes lithium deintercalation more difficult. 


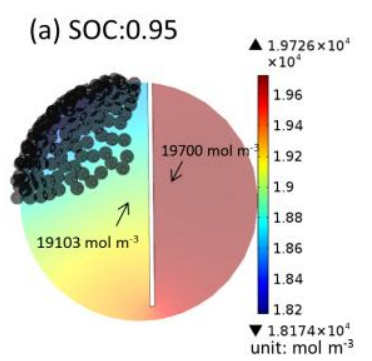

$\Delta c=597 \mathrm{~mol} \mathrm{~m}^{-3}$ (b) SOC:0.45

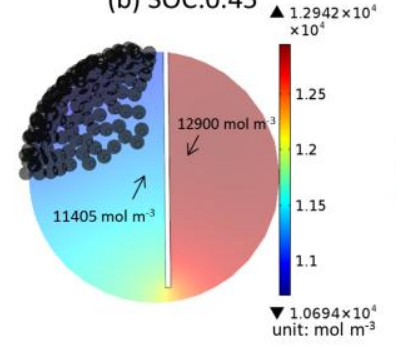

$\Delta c=1495 \mathrm{~mol} \mathrm{~m}^{-3}$ (c) SOC:0.15

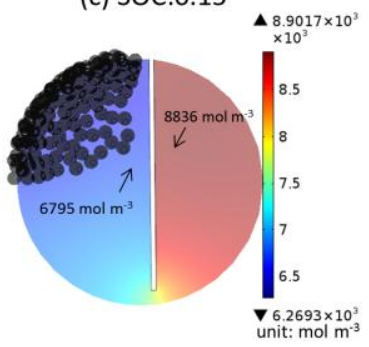

$\Delta c=2041 \mathrm{~mol} \mathrm{~m}^{-3}$

(d) Ionic conductivity and electronic conductivity

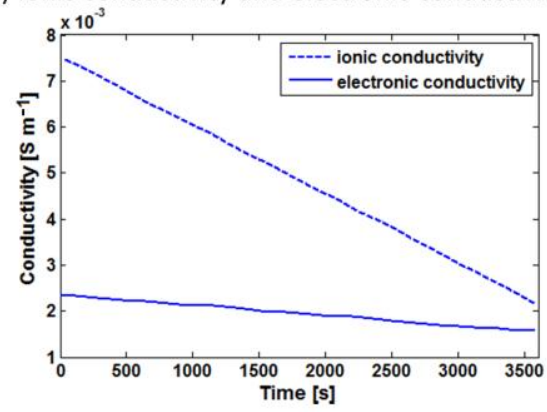

Figure 8. (a)-(c) Cross-section view of lithium ion concentration inside particles at various SOCs. Higher SOC reduces the impact from fracture. Particle diameter: $5 \mu \mathrm{m}$. (d) Comparison between ionic conductivity and electronic conductivity.

Our simulations suggest that higher SOC is preferable to suppress the impact of fracture. Higher SOC brings both high electronic conductivity and ionic conductivity.

\section{Conclusions}

We have developed a particle model to study the impact of fracture on capacity degradation. This model considers realistic anisotropic environment surrounding an active particle and incorporates both diffusion and migration of lithium ions and electrons in the particle. We have applied the model to investigate the impact of conductivity, the SOC swing window, and particle size on fracture-induced capacity degradation.

The study shows that electronic conductivity has a significant effect on lithium ion distribution in the particle. Electronic conductivity, when it is low, is the main limiting factor on 
lithium deintercalation/intercalation. As electronic conductivity increases, the limiting factor shifts from electron transport to lithium transport. When the electronic conductivity is high enough, lithium transport can be assumed to be diffusion-only. Only under this situation the assumption used by the conventional model, i.e. isotropic environment and diffusion controlled lithium ion transport, is valid. Still, the existence of fracture easily breaks the assumption. When fracture is present, the resistance for electron transport increases inside a spherical particle and the transport becomes highly unsymmetrical and location-dependent. Lithium ions get stuck and cannot deintercalate or intercalate, leading to reduced capacity.

SOC has a significant impact on conductivity and capacity degradation associated with fracture. When the SOC is high, the particle is full of lithium and electrons, and both electronic conductivity and ionic conductivity are relatively high. Therefore, the electrons can be pulled out relatively easily from a cathode particle during charging. The SOC study suggests that the cathode should be run at a higher SOC level to gain good conductivity to suppress the impact of fracture on capacity degradation. Particle size is another key factor that affects lithium ion transport in the particle. A larger particle size leads to higher internal resistance for electron transport; therefore, fracture-induced capacity degradation is more severe in comparison to that in a particle of smaller size. In order to suppress the impact associated with fracture, smaller particle size is preferable.

\section{Acknowledgments}

This work was supported by the National Science Foundation under Grant No. CNS1446117. 


\section{Appendix}

The electrochemical potential is the driving force for the flux of species in the electrolyte. The flux density of mobile species in the electrolyte can be expressed by [18]

$$
\begin{aligned}
& \mathbf{J}_{3,+}=-\frac{v_{3,+} D_{3}}{v R T} \frac{c_{T}}{c_{0}} c_{3} \nabla \mu_{2}+\frac{\mathbf{i} t_{+}^{0}}{z_{3,+} F}+c_{3,+} v_{0} \\
& \mathbf{J}_{3,-}=-\frac{v_{3,-} D_{3}}{v R T} \frac{c_{T}}{c_{0}} c_{3} \nabla \mu_{2}+\frac{\mathbf{i} t_{-}^{0}}{z_{3,-} F}+c_{3,-} v_{0}
\end{aligned}
$$

where $\mu_{2}=v R T \ln \left(c_{3} f_{ \pm} a_{ \pm}^{\theta}\right)$ is the electrochemical potential of the electrolyte, $v=v_{3,+}+v_{3,-}$, and $a_{ \pm}^{\theta}$ is a proportional constant. The last term is negligible when the solvent velocity, $v_{0}$, is small.

The current density is given by [18]

$$
\mathbf{i}=-k_{3} \nabla \phi_{3}+k_{3} \frac{2 R T}{c_{3} F}\left(1+\frac{d \ln f_{ \pm}}{d \ln c_{3}}\right)\left(1-t_{+}^{0}\right) \nabla c_{3}
$$

In order to express species flux densities in terms of concentration gradient and potential gradient, Eq. (A3) is substituted into Eqs.(A1) and (A2) to read:

$$
\begin{aligned}
& \mathbf{J}_{3,+}=-v_{3,+} D_{3} \frac{c_{T}}{c_{0}}\left(1+\frac{d \ln f_{ \pm}}{d \ln c_{3}}\right) \nabla c_{3}+\frac{\mathbf{i} t_{+}^{0}}{z_{3,+} F} \\
& =-v_{3,+} D_{3} \frac{c_{T}}{c_{0}}\left(1+\frac{d \ln f_{ \pm}}{d \ln c_{3}}\right) \nabla c_{3}+\frac{2 k_{3} t_{+}^{0} R T}{c_{3} z_{3,+} F^{2}}\left(1+\frac{d \ln f_{ \pm}}{d \ln c_{3}}\right)\left(1-t_{+}^{0}\right) \nabla c_{3}-\frac{k_{3} t_{+}^{0}}{z_{3,+} F} \nabla \phi_{3} \\
& =\left(-v_{3,+} D_{3} \frac{c_{T}}{c_{0}}+\frac{2 k_{3} t_{-}^{0} t_{+}^{0} R T}{c_{3} z_{3,+} F^{2}}\right)\left(1+\frac{d \ln f_{ \pm}}{d \ln c_{3}}\right) \nabla c_{3}-\frac{k_{3} t_{+}^{0}}{z_{3,+} F} \nabla \phi_{3} \\
& =\left(-D_{3} \frac{c_{T}}{c_{0}}+\frac{2 k_{3} t_{-}^{0} t_{+}^{0} R T}{c_{3,+} z_{3,+} F^{2}}\right)\left(1+\frac{d \ln f_{ \pm}}{d \ln c_{3}}\right) \nabla c_{3,+}-\frac{k_{3} t_{+}^{0}}{z_{3,+} F} \nabla \phi_{3}
\end{aligned}
$$

Similarly

$$
\mathbf{J}_{3,-}=\left(-D_{3} \frac{c_{T}}{c_{0}}+\frac{2 k_{3} t_{-}^{0} t_{-}^{0} R T}{c_{3,-} z_{3,-} F^{2}}\right)\left(1+\frac{d \ln f_{ \pm}}{d \ln c_{3}}\right) \nabla c_{3,-}-\frac{k_{3} t_{-}^{0}}{z_{3,-} F} \nabla \phi_{3}
$$




\section{Nomenclature}

\begin{tabular}{|c|c|}
\hline$c_{0}$ & solvent concentration, $\mathrm{mol} \mathrm{m}^{-3}$ \\
\hline$c_{2, \mathrm{e}}$ & concentration of electron in particle, $\mathrm{mol} \mathrm{m}^{-3}$ \\
\hline$c_{2, \mathrm{Li}}$ & concentration of lithium ion in particle, $\mathrm{mol} \mathrm{m}^{-3}$ \\
\hline$c_{2, \mathrm{Mn}_{2} \mathrm{O}_{4}}$ & concentration of $\left(\mathrm{Mn}_{2} \mathrm{O}_{4}\right)^{x+}$ ion in particle, mol m${ }^{-3}$ \\
\hline$c_{3}$ & electrolyte concentration, $\mathrm{mol} \mathrm{m}^{-3}$ \\
\hline$c_{3,+}$ & concentration of lithium ion in electrolyte, $\mathrm{mol} \mathrm{m}^{-3}$ \\
\hline$c_{3,-}$ & concentration of negative ion in electrolyte, $\mathrm{mol} \mathrm{m}^{-3}$ \\
\hline$c_{T}$ & total concentration including the solvent, $\mathrm{mol} \mathrm{m}^{-3}$ \\
\hline$D_{2, \mathrm{e}}$ & diffusivity of electron in particle, $\mathrm{m}^{2} \mathrm{~s}^{-1}$ \\
\hline$D_{2, \mathrm{Li}}$ & diffusivity of lithium ion in particle, $\mathrm{m}^{2} \mathrm{~s}^{-1}$ \\
\hline$D_{2, \mathrm{Mn}_{2} \mathrm{O}_{4}}$ & diffusivity of $\left(\mathrm{Mn}_{2} \mathrm{O}_{4}\right)^{x+}$ ion in particle, which is very small, $\mathrm{m}^{2} \mathrm{~s}^{-1}$ \\
\hline$D_{3}$ & diffusivity of electrolyte, $\mathrm{m}^{2} \mathrm{~s}^{-1}$ \\
\hline $\mathbf{E}_{1}$ & electric field in the carbon black network, $\mathrm{V} \mathrm{m}^{-1}$ \\
\hline$f_{ \pm}$ & electrolyte activity coefficient \\
\hline$F$ & Faraday constant, $\mathrm{C} \mathrm{mol}^{-1}$ \\
\hline $\mathbf{i}_{1}$ & current density in the carbon black network, $\mathrm{A} \mathrm{m}^{-2}$ \\
\hline $\mathbf{i}_{2}$ & current density in particle, $\mathrm{A} \mathrm{m}^{-2}$ \\
\hline $\mathbf{i}_{3}$ & current density in electrolyte, $\mathrm{A} \mathrm{m}^{-2}$ \\
\hline$i_{23}$ & reaction current density between particle and electrolyte, $\mathrm{A} \mathrm{m}^{-2}$ \\
\hline$i_{23,0}$ & exchange current density between particle and electrolyte, $\mathrm{A} \mathrm{m}^{-2}$ \\
\hline$i_{43}$ & reaction current between lithium metal anode and electrolyte, $\mathrm{A} \mathrm{m}^{-2}$ \\
\hline$i_{43,0}$ & exchange current density between lithium metal anode and electrolyte, $\mathrm{A} \mathrm{m}^{-2}$ \\
\hline $\mathbf{J}_{2, \mathrm{e}}$ & flux density of electron in particle, $\mathrm{mol} \mathrm{m}^{-2} \mathrm{~s}^{-1}$ \\
\hline $\mathbf{J}_{2, \mathrm{Li}}$ & flux density of lithium ion in particle, $\mathrm{mol} \mathrm{m}^{-2} \mathrm{~s}^{-1}$ \\
\hline $\mathbf{J}_{2, \mathrm{Mn}_{2} \mathrm{O}_{4}}$ & flux density of $\left(\mathrm{Mn}_{2} \mathrm{O}_{4}\right)^{x+}$ ion in particle, which is zero, $\mathrm{mol} \mathrm{m}^{-2} \mathrm{~s}^{-1}$ \\
\hline $\mathbf{J}_{3,+}$ & flux density of lithium ion in electrolyte, $\mathrm{mol} \mathrm{m}^{-2} \mathrm{~s}^{-1}$ \\
\hline $\mathbf{J}_{3,-}$ & flux density of anion in electrolyte, $\mathrm{mol} \mathrm{m}^{-2} \mathrm{~s}^{-1}$ \\
\hline$k_{2}$ & conductivity of particle, $\mathrm{S} \mathrm{m}^{-1}$ \\
\hline$k_{3}$ & conductivity of electrolyte, $\mathrm{S} \mathrm{m}^{-1}$ \\
\hline$R$ & gas constant, $\mathrm{J} \mathrm{mol}^{-1} \mathrm{~K}^{-1}$ \\
\hline$t_{+}^{0}$ & transference number of lithium ion in electrolyte \\
\hline$t_{-}^{0}$ & transference number of negative ion in electrolyte \\
\hline$T$ & absolute temperature, $\mathrm{K}$ \\
\hline
\end{tabular}




$\begin{array}{cl}u_{m, i} & \text { mobility of species } i \text { in particle, mol s kg-1 } \\ U & \text { cathode equilibrium potential, } \mathrm{V} \\ \alpha_{a} & \text { anodic charge transfer coefficient between particle and electrolyte } \\ \alpha_{c} & \text { cathodic charge transfer coefficient between particle and electrolyte } \\ \alpha_{a, \mathrm{Li}} & \text { anodic charge transfer coefficient between lithium metal anode and electrolyte } \\ \alpha_{c, \mathrm{Li}} & \text { cathodic charge transfer coefficient between lithium metal anode and electrolyte } \\ v_{3,+} & \text { number of cation per molecular of electrolyte } \\ v_{3,-} & \text { number of anion per molecular of electrolyte } \\ z_{2, \mathrm{e}} & \text { charge number of electron } \\ z_{2, \mathrm{Li}} & \text { charge number of lithium ion } \\ z_{2, \mathrm{Mn} \mathrm{O}_{4}} & \left.\text { charge number of (Mn } \mathrm{O}_{4}\right)^{x+} \text { ion } \\ z_{3,+} & \text { charge number of cation in electrolyte } \\ z_{3,-} & \text { charge number of anion in electrolyte } \\ \sigma_{1} & \text { conductivity of carbon black, } \mathrm{S} \text { m } \\ \eta_{23} & \text { over potential between particle and electrolyte, } \mathrm{V} \\ \eta_{43} & \text { over potential between lithium metal and electrolyte, } \mathrm{V} \\ \phi_{1} & \text { electric potential in the carbon black network, } \mathrm{V} \\ \phi_{2} & \text { electric potential in particle, } \mathrm{V} \\ \phi_{3} & \text { electric potential in electrolyte, } \mathrm{V} \\ \phi_{4} & \text { electric potential of lithium metal anode, } \mathrm{V} \\ & \end{array}$


Table 1 Values of model parameters

\begin{tabular}{|c|c|c|c|}
\hline Paramters & Description & Value & Ref. \\
\hline$\sigma_{1}\left(\mathrm{~S} \mathrm{~m}^{-1}\right)$ & Conductivity of carbon black & 100 & {$[21]$} \\
\hline$c_{2, \mathrm{Li}}^{\max }\left(\mathrm{mol} \mathrm{m}^{-3}\right)$ & Maximum solid phase concentration & 22860 & {$[15]$} \\
\hline$k_{0}\left(\mathrm{~m} \mathrm{~s}^{-1}\right)$ & Reaction rate coefficient & $1 \times 10^{-11}$ & [19] \\
\hline$\alpha_{a}, \alpha_{c}$ & $\begin{array}{l}\text { Charge transfers coefficient between LMO } \\
\text { particle and electrolyte }\end{array}$ & 0.5 & {$[15]$} \\
\hline$i_{23,0}\left(\mathrm{~A} \mathrm{~m}^{-2}\right)$ & $\begin{array}{l}\text { Exchange current density between LMO and } \\
\text { electrolyte }\end{array}$ & $F k_{0} c_{3}^{\alpha_{a}}\left(c_{2, \mathrm{Li}}^{\max }-c_{2, \mathrm{Li}}\right)^{\alpha_{a}} c_{2}^{\alpha_{c}}$ & {$[15]$} \\
\hline$U(\mathrm{~V})$ & LMO cathode equilibrium potential & measured curve & [5] Fig A-2 \\
\hline$D_{2, \mathrm{Li}}\left(\mathrm{m}^{2} \mathrm{~s}^{-1}\right)$ & Diffusivity of lithium ion in particle & $1 \times 10^{-13}$ & {$[15]$} \\
\hline$D_{2, \mathrm{e}}\left(\mathrm{m}^{2} \mathrm{~s}^{-1}\right)$ & Diffusivity of electron in particle & $1.5 \times 10^{-14}$ & fitted \\
\hline$D_{2, \mathrm{Mn}_{2} \mathrm{O}_{4}}\left(\mathrm{~m}^{2} \mathrm{~s}^{-1}\right)$ & Diffusivity of $\left(\mathrm{Mn}_{2} \mathrm{O}_{4}\right)^{x+}$ ion in particle & $1 \times 10^{-900}$ & \\
\hline$z_{2, \mathrm{Li}}$ & Charge number of lithium ion & 1 & \\
\hline$z_{2, \mathrm{e}}$ & Charge number of electron & -1 & \\
\hline$z_{2, \mathrm{Mn}_{2} \mathrm{O}_{4}}$ & Charge number of $\left(\mathrm{Mn}_{2} \mathrm{O}_{4}\right)^{x+}$ ion & 0.875 & fitted \\
\hline$v_{3,+}$ & Number of cations per molecular of electrolyte & 1 & \\
\hline$v_{3,-}$ & Number of anions per molecular of electrolyte & 1 & \\
\hline$c_{0}\left(\mathrm{~mol} \mathrm{~m}^{-3}\right)$ & Solvent concentration & 2070 & {$[22]$} \\
\hline$c_{3}\left(\mathrm{~mol} \mathrm{~m}^{-3}\right)$ & Electrolyte concentration & 1000 & experiment \\
\hline$c_{T}\left(\mathrm{~mol} \mathrm{~m}^{-3}\right)$ & Total concentration including the solvent & 4070 & Eq. (15) \\
\hline$D_{3}\left(\mathrm{~m}^{2} \mathrm{~s}^{-1}\right)$ & Diffusivity of electrolyte & $1.27 \times 10^{-11}$ & {$[20]$} \\
\hline$k_{3}\left(\mathrm{~S} \mathrm{~m}^{-1}\right)$ & Conductivity of electrolyte & 0.38 & {$[23]$} \\
\hline$t_{+}^{0}$ & Lithium ion transference number & 0.363 & {$[15]$} \\
\hline$t_{-}^{0}$ & Anion transference number & $1-t_{+}^{0}$ & \\
\hline$z_{3,+}$ & Charge number of cation in electrolyte & 1 & \\
\hline$z_{3,-}$ & Charge number of anion in electrolyte & -1 & \\
\hline$f_{ \pm}$ & Electrolyte activity coefficient & 1 & \\
\hline$i_{43,0}\left(\mathrm{~A} \mathrm{~m}^{-2}\right)$ & $\begin{array}{l}\text { Exchange current density between lithium metal } \\
\text { and electrolyte }\end{array}$ & 8500 & {$[20]$} \\
\hline$\alpha_{a, \mathrm{Li}}, \alpha_{c, \mathrm{Li}}$ & Charge transfer coefficient & 0.5 & {$[20]$} \\
\hline
\end{tabular}




\section{References}

[1] S. Eben Li, K. Li, J. Wang, Vehicle System Dynamics, 51 (2013) 1-17.

[2] S.E. Li, H. Peng, K. Li, J. Wang, IEEE Transactions on Vehicular Technology, 61 (2012) 998-1007.

[3] X. Lin, A. Ivanco, Z. Filipi, SAE International Journal of Alternative Powertrains, 1 (2012) 249-259.

[4] M. Woon, X. Lin, A. Ivanco, A. Moskalik, C. Gray, Z. Filipi, SAE Technical Paper, (2011) 01-0868.

[5] X. Lin, J. Park, L. Liu, Y. Lee, A.M. Sastry, W. Lu, Journal of The Electrochemical Society, 160 (2013) A1701-A1710.

[6] B. Wu, W. Lu, Journal of the Electrochemical Society, 163 (2016) A3131-A3139.

[7] J. Park, W. Lu, A.M. Sastry, Journal of the Electrochemical Society, 158 (2011) A201-A206.

[8] T.M. Bandhauer, S. Garimella, T.F. Fuller, Journal of the Electrochemical Society, 158 (2011) R1-R25.

[9] A. Barre, B. Deguilhem, S. Grolleau, M. Gerard, F. Suard, D. Riu, Journal of Power Sources, 241 (2013) 680-689.

[10] M.-R. Lim, W.-I. Cho, K.-B. Kim, Journal of Power Sources, 92 (2001) 168-176.

[11] X. Zhang, W. Shyy, A.M. Sastry, Journal of the Electrochemical Society, 154 (2007) A910A916.

[12] M. Zhu, J. Park, A.M. Sastry, Journal of The Electrochemical Society, 159 (2012) A492A498.

[13] A. Blyr, C. Sigala, G. Amatucci, D. Guyomard, Y. Chabre, J.M. Tarascon, Journal of The Electrochemical Society, 145 (1998) 194-209. 
[14] H. Shin, J. Park, A.M. Sastry, W. Lu, Journal of Power Sources, 284 (2015) 416-427.

[15] M. Doyle, J. Newman, A.S. Gozdz, C.N. Schmutz, J.M. Tarascon, Journal of The

Electrochemical Society, 143 (1996) 1890-1903.

[16] D. Wang, X. Wu, Z. Wang, L. Chen, Journal of Power Sources, 140 (2005) 125-128.

[17] S. Yamamura, H. Koshika, M. Nishizawa, T. Matsue, I. Uchida, Journal of Solid State Electrochemistry, 2 (1998) 211-215.

[18] J. Newman, K.E. Thomas-Alyea, Electrochemical Systems, 3rd ed., Wiley-Interscience, (2004).

[19] C.M. Doyle, Design and simulation of lithium rechargeable batteries, $\mathrm{PhD}$ Thesis, University of California, Berkeley, (1995).

[20] C.-W. Wang, A.M. Sastry, Journal of The Electrochemical Society, 154 (2007) A1035A1047.

[21] B. Marinho, M. Ghislandi, E. Tkalya, C.E. Koning, G. de With, Powder Technol, 221 (2012) $351-358$.

[22] J.P. Cho, B. Park, Journal of Power Sources, 92 (2001) 35-39.

[23] A. Vallee, S. Besner, J. Prudhomme, Electrochim Acta, 37 (1992) 1579-1583. 

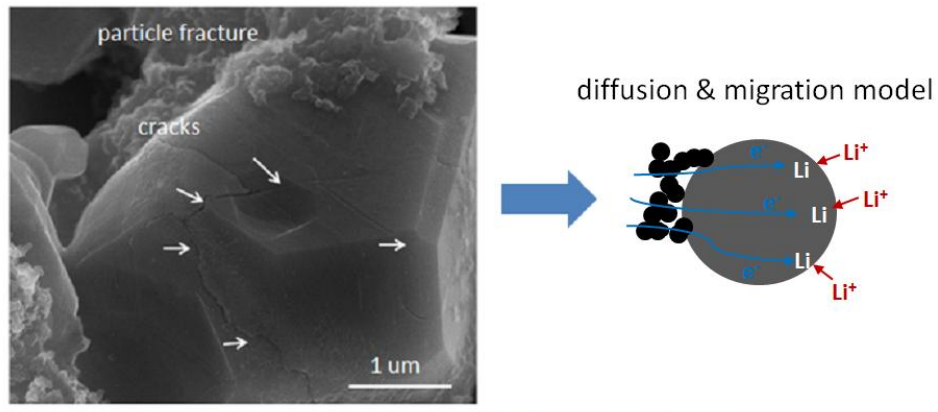

Lithium ion distribution without fracture

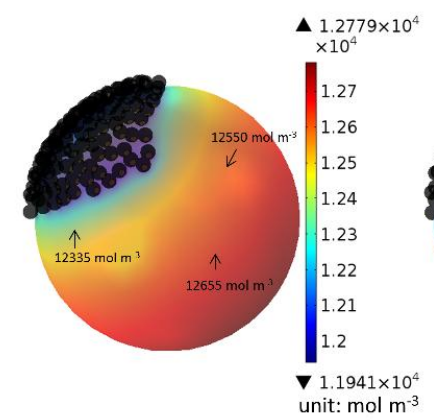

Lithium ion distribution with fracture

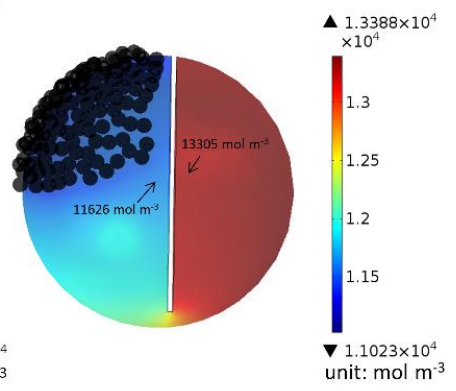

